Zürich University Preprint

ZU-TH 33/96

gr-qc/9703052

\title{
Time evolution of the perturbations for a complex scalar field in a Friedmann-Lemaître universe*
}

\author{
Philippe Jetzer ${ }^{1,2}$ and David Scialom ${ }^{2}$ \\ ${ }^{1}$ Paul Scherrer Institute, Laboratory for Astrophysics, CH-5232 Villigen \\ PSI \\ 2 Institute of Theoretical Physics, University of Zürich, \\ Winterthurerstrasse 190, CH-8057 Zürich, Switzerland
}

\begin{abstract}
We study the time evolution of small classical perturbations in a gauge invariant way for a complex scalar field in the early zero curvature Friedmann-Lemaitre universe. We, thus, generalize the analysis which has been done so far for a real scalar field. We give also a derivation of the Jeans wavenumber in the Newtonian regime starting from the general relativistic equations, avoiding the so-called Jeans swindle.

During the inflationary phase, whose length depends on the value of the bosonic charge, the behavior of the perturbations turns out to be the same as for a real scalar field. In the oscillatory phase the time evolution of the perturbations can be determined analytically as long as the bosonic charge of the corresponding background solution is sufficiently large. This is not possible for the real scalar field, since the corresponding bosonic charge vanishes.
\end{abstract}

PACS number(s): 98.80.Cq, 98.80.Hw

* This work was partially supported by the Swiss National Science Foundation. 


\section{Introduction}

The developments in particle physics and cosmology suggest that scalar fields may have played an important role in the evolution of the early universe, for instance in primordial phase transitions, and that they may constitute part of the dark matter. Moreover, scalar fields are an important ingredient in most of the particle physics models based on the unification of the fundamental forces, as for instance in superstring theories. Scalar particles are needed in cosmological models based on inflation, whose relevance is supported by the results of the COBE-DMR measurements that are consistent with an Harrison-Zel'dovich spectrum [1].

These facts, in particular inflation, motivated the study of the coupled Einstein-scalar field equations to determine the time evolution and also the gravitational equilibrium configurations of scalar fields, which may form socalled boson stars [2, 3].

A detailed study of the solutions of the Einstein equations for a homogeneous isotropic Friedmann-Lemaitre universe with a real scalar field has been carried out in particular by Belinsky et al. [1, 5, 6], Piran and Williams [7]. The corresponding thorough investigation for a complex scalar field has been performed in Ref. 8

The knowledge of the behavior of the background solution in the early universe is of importance for the analysis of the time evolution of the perturbations [9, 10]. This is of relevance if scalar fields make up part of the dark matter and form compact objects, such as boson stars, or trigger the formation of the observed large scale structures in the universe.

In this paper we study the time evolution of small perturbations in a gauge invariant way for a complex scalar field in the early zero curvature Friedmann-Lemaitre universe thus, generalizing the previous analysis for a real scalar field [11]. We consider a potential with a positive mass and a quartic self-interaction term and closely follow the treatment developed by Mukhanov et al. [10]. During the inflationary phase the behavior of the perturbations is similar to the one of the real scalar field. This is not surprising, since along the separatrices the phase of the complex scalar field remains constant and thus inflation is essentially driven by one component of the field [8]. The short wavelength perturbations get smeared out, whereas the long wavelength perturbations increase. On the other hand, the metric long wavelength perturbations are entirely determined by the scale factor and hence survive the decay of the complex scalar field background solution after inflation.

We extend our investigation of the behavior of the perturbations also to the oscillatory phase, which takes place after inflation. Contrary to the real 
scalar field for which the bosonic charge of the background solution vanishes, the charge is different from zero in our case. If it is sufficiently large, we can even find analytically the time evolution of the perturbations for a massive complex scalar field. It turns out, that the long wavelength perturbations decrease with time, whereas the short wavelength perturbations, at best, oscillate around a constant value. Therefore, we do not expect the formation of gravitational seeds around which structure can form for the case of a massive complex scalar field with a sufficiently large bosonic charge. The other cases have to be treated numerically. In order to put constraints on the parameters of the scalar field potential from the measured cosmic microwave background (CMB) anisotropies [1], we have to analyze the quantized perturbations. Here, we have restricted the analysis only to the classical perturbations.

Moreover, we also derive the Jeans wavenumber in the Newtonian regime starting from the general relativistic equations, thus avoiding the so-called "Jeans swindle". For the case where there is a quartic self-interaction term in the potential the result we find differs from the one given in Ref. [12].

The paper is organized as follows. In section 2 we present the basic equations, which we will use. In section 3 we give the derivation of the Jeans wavenumber starting from the general relativistic equations. Section to devoted to the analysis of the time evolution of the classical scalar mode fluctuations in a fully general relativistic way, both in the inflationary and in the oscillatory phases. The numerical results are presented in section 5 and in section 6 we present briefly our conclusions.

\section{Basic equations}

We will consider the linear scalar mode perturbations since they are the only ones which contribute to the energy density fluctuations [13. As usual, we expand the scalar perturbations in terms of a complete set of harmonic functions $Y_{k}$, which are the eigenfunctions with eigenvalue $-k^{2}$ of the LaplaceBeltrami operator $\Delta$ defined on constant time slices $\Sigma_{\eta}$. In the following, we will omit for simplicity to write the subscript $k$ from $Y$.

The zero curvature Friedmann-Lemaitre metric - including the first order perturbations - is then given by 13

$$
d s^{2}=g_{\mu \nu} \theta^{\mu} \theta^{\nu}=-\theta^{0} \theta^{0}+\delta_{i j} \theta^{i} \theta^{j}
$$


where

$$
\begin{gathered}
\theta^{0}=\alpha d \eta=a(1+A Y) d \eta \\
\theta^{i}=\beta^{i} d \eta+a\left[\left(1+H_{L} Y\right) d x^{i}+H_{T} Y_{j}^{i} d x^{j}\right] \\
=-a B Y^{i} d \eta+a\left[\left(1+H_{L} Y\right) d x^{i}+H_{T} Y_{j}^{i} d x^{j}\right],
\end{gathered}
$$

and

$$
Y_{i}=-k^{-1} Y_{\mid i}, \quad Y_{i j}=k^{-2} Y_{\mid i j}+\frac{1}{3} Y \delta_{i j} .
$$

$\alpha$ is the lapse function and $\beta^{i}$ are the components of the shift vector. $A$, $B, H_{L}$ and $H_{T}$ are, respectively, the amplitude of the perturbation of the lapse function, the shift vector, the unit spatial volume and the anisotropic distortion of $\Sigma_{\eta}$. All these functions, as well as the scale factor $a$, depend only on the conformal time $\eta$. The subscript ${ }_{\mid i}$ denotes the covariant derivative on $\left(\Sigma_{\eta}, \gamma\right)$, where $a^{2} \gamma$ is defined as the unperturbed induced metric on $\Sigma_{\eta}$. It is easy to verify that $Y_{i j}$ is traceless.

We take for the matter action

$$
S_{m}=\int \zeta\left[\frac{1}{2} g^{\mu \nu}\left(e_{\mu} \Phi e_{\nu} \Phi^{*}+e_{\nu} \Phi e_{\mu} \Phi^{*}\right)+V\left(\Phi, \Phi^{*}\right)\right],
$$

where $\zeta$ is the volume form and $e_{\mu}$ is the dual basis of $\theta^{\mu}$. We expand the complex scalar field $\Phi$ to first order, where $\phi(\eta)$ is the zero-order background solution and $\delta \phi(\eta) Y$ is the first order term. The potential $V$ is such that $S_{m}$ is invariant under a global $U(1)$ symmetry. Thus, V has to fulfill the following conditions

$$
\begin{aligned}
\phi \frac{\partial V}{\partial \phi}-\phi^{*} \frac{\partial V}{\partial \phi^{*}} & =0, \\
\phi \frac{\partial V}{\partial \phi}+\phi^{2} \frac{\partial^{2} V}{\partial \phi^{2}}-\phi \phi^{*} \frac{\partial^{2} V}{\partial \phi \partial \phi^{*}} & =0, \\
\phi^{*} \frac{\partial V}{\partial \phi^{*}}+\phi^{* 2} \frac{\partial^{2} V}{\partial \phi^{* 2}}-\phi \phi^{*} \frac{\partial^{2} V}{\partial \phi \partial \phi^{*}} & =0 .
\end{aligned}
$$

The $U(1)$-global symmetry of $S_{m}$ implies a conserved current

$$
J=J^{(0)}+\delta J
$$

where

$$
\begin{aligned}
J^{(0)}= & -\frac{i}{a}\left(\dot{\phi}^{*} \phi-\dot{\phi} \phi^{*}\right) e_{0}, \\
\delta J= & \left\{-\frac{i}{a}\left[\dot{\phi}^{*} \delta \phi-\dot{\phi} \delta \phi^{*}+\phi \dot{\delta \phi} \dot{\phi}^{*} \phi^{*} \dot{\delta \phi}\right]-J^{(0)} A\right\} Y e_{0} \\
& -\frac{i k}{a}\left[\phi \delta \phi^{*}-\phi^{*} \delta \phi\right] Y^{i} e_{i} .
\end{aligned}
$$


Dot means the derivative with respect to $\eta$. By varying $S_{m}$ with respect to the metric, we obtain the energy-momentum tensor up to the first order terms

$$
T=\varepsilon e_{0} \otimes e_{0}+e_{0} \otimes \mathbf{S}+\mathbf{S} \otimes e_{0}+\mathbf{T} \equiv T^{(0)}+\delta T,
$$

where

$$
\begin{aligned}
\varepsilon \equiv & \varepsilon^{(0)}+\delta \varepsilon \\
= & \frac{1}{a^{2}}\left[\dot{\phi}^{*} \dot{\phi}+a^{2} V\right. \\
+ & \left.\dot{\phi}^{*} \dot{\delta \phi}+\dot{\phi} \dot{\delta \phi}-2 A Y \dot{\phi}^{*} \dot{\phi}+a^{2} \frac{\partial V}{\partial \phi} \delta \phi+a^{2} \frac{\partial V}{\partial \phi^{*}} \delta \phi^{*}\right] \\
\mathbf{S} \equiv & \mathbf{S}^{(0)}+\delta \mathbf{S}=\frac{k}{a^{2}}\left(\dot{\phi} \delta \phi^{*}+\dot{\phi}^{*} \delta \phi\right) Y^{i} e_{i}, \\
\mathbf{T} \equiv & \mathbf{T}^{(0)}+\delta \mathbf{T} \\
= & \frac{1}{a^{2}}\left[\dot{\phi} \dot{\phi}^{*}-a^{2} V+\left(\dot{\phi} \dot{\delta \phi}{ }^{*}+\dot{\phi}^{*} \dot{\delta \phi}-2 A \dot{\phi} \dot{\phi}^{*}\right.\right. \\
& \left.\left.-a^{2} \frac{\partial V}{\partial \phi} \delta \phi-a^{2} \frac{\partial V}{\partial \phi^{*}} \delta \phi^{*}\right) Y\right] \delta^{i j} e_{i} \otimes e_{j} .
\end{aligned}
$$

The background quantities and the first order perturbation components can be easily identified. To derive the Einstein equation using the $3+1$ formalism [14], we first have to compute the second fundamental form $\mathbf{K}$ for the slices $\Sigma_{\eta}$, for which we get

$$
\mathbf{K}=-\frac{1}{a}\left[h \delta^{i j}+k \sigma_{g} Y^{i j}+h \kappa_{g} Y \delta^{i j}\right] e_{i} \otimes e_{j},
$$

where $h=\frac{\dot{a}}{a}$ is the Hubble expansion rate,

$$
\sigma_{g}=\frac{\dot{H}_{T}}{k}-B
$$

and

$$
\kappa_{g}=-A+\frac{k B}{3 h}+\frac{\dot{H}_{L}}{h} .
$$


The Einstein equations are then given by 15

$$
\begin{gathered}
\mathbf{R}+[\operatorname{Tr}(\mathbf{K})]^{2}-\operatorname{Tr}\left(\mathbf{K}^{2}\right)=16 \pi G \varepsilon, \\
\nabla \cdot \mathbf{K}-\nabla \operatorname{Tr} \mathbf{K}=8 \pi G \mathbf{S}, \\
\partial_{t} \mathbf{K}=\mathbf{L}_{\beta} \mathbf{K}-\mathbf{H e s s}(\alpha)+\alpha\left[\mathbf{R i c}(\mathbf{g})+2 \mathbf{K}^{2}\right. \\
+\mathbf{K} \operatorname{Tr} \mathbf{K}-8 \pi G \mathbf{T}-4 \pi G \mathbf{g}(\varepsilon-\operatorname{Tr} \mathbf{T})],
\end{gathered}
$$

where Tr denotes the trace, Hess the Hessian, $\mathbf{g}$ the induced metric on $\Sigma_{\eta}$, $\mathbf{R}$ and $\mathbf{R i c}(\mathbf{g})$ are, respectively, the Ricci scalar and the Ricci tensor of the slices $\Sigma_{\eta}$. The background solution trivially satisfies eq.(15), whereas from eq.(14) and eq.(16) we obtain

$$
\begin{aligned}
3 h^{2} & =8 \pi G\left[\dot{\phi} \dot{\phi}^{*}+a^{2} V\right], \\
\dot{h}+2 h^{2} & =8 \pi G a^{2} V .
\end{aligned}
$$

¿From eq.(16) we get for the terms in first order

$$
\begin{aligned}
k^{2} Y_{i j}\left(\Phi_{g}+\psi\right)= & -Y \delta_{i j}\left[-\ddot{\psi}-5 h \dot{\psi}-\frac{4}{3} k^{2} \psi\right. \\
& +h \dot{\Phi}_{g}+\left(16 \pi G a^{2} V-\frac{k^{2}}{3}\right) \Phi_{g} \\
& \left.+8 \pi G a^{2}\left(\frac{\partial V}{\partial \phi} \delta \varphi+\frac{\partial V}{\partial \phi^{*}} \delta \varphi^{*}\right)\right],
\end{aligned}
$$

where we have introduced the gauge invariant quantities $[16,13,10$

$$
\begin{aligned}
\psi & =H_{L}+\frac{1}{3} H_{T}-\frac{1}{k} h \sigma_{g}, \\
\delta \varphi & =\delta \phi-\dot{\phi} \frac{\sigma_{g}}{k}, \\
\Phi_{g} & =A-\frac{1}{k a}\left(a \sigma_{g}\right) .
\end{aligned}
$$

For $i \neq j$ we obtain

$$
\psi=-\Phi_{g}
$$

The meaning of the gauge invariant quantities is obvious on small scales (i.e. $k \gg 1$ ), in which case they reduce to $H_{L}+\frac{1}{3} H_{T}$ (the intrinsic scalar curvature perturbation), $\delta \phi$ (the complex scalar field perturbation) and $A$ (the gravitational potential), respectively. 
The first order terms of eqs.(14)-(15) combined with the trace of eq.(19) lead to the following Einstein equations

$$
\begin{aligned}
6 h \dot{\psi}+\left(2 k^{2}+6 h^{2}\right) \psi= & 8 \pi G\left(\dot{\phi}^{*} \dot{\delta} \varphi+\dot{\phi} \dot{\delta} \varphi^{*}+2 \dot{\phi} \dot{\phi}^{*} \psi\right. \\
& \left.+a^{2} \frac{\partial V}{\partial \phi} \delta \varphi+a^{2} \frac{\partial V}{\partial \phi^{*}} \delta \varphi^{*}\right) \\
\dot{\psi}+h \psi= & -4 \pi G\left(\dot{\phi}^{*} \delta \varphi+\dot{\phi} \delta \varphi^{*}\right) \\
\ddot{\psi}+6 h \dot{\psi}+\left(k^{2}+16 \pi G a^{2} V\right) \psi= & 8 \pi G a^{2}\left(\frac{\partial V}{\partial \phi} \delta \varphi+\frac{\partial V}{\partial \phi^{*}} \delta \varphi^{*}\right)
\end{aligned}
$$

where we used eq.(23).

By varying $S_{m}$ with respect to $\Phi^{*}$ we obtain the Klein-Gordon equation, which to the zero order is given by

$$
\ddot{\phi}+2 h \dot{\phi}+a^{2} \frac{\partial V}{\partial \phi^{*}}=0
$$

By directly expressing the first order perturbed Klein-Gordon equation in terms of the above defined gauge invariant quantities, we get

$$
\ddot{\delta} \varphi+2 h \dot{\delta} \varphi+k^{2} \delta \varphi+4 \dot{\phi} \dot{\psi}-2 a^{2} \frac{\partial V}{\partial \phi^{*}} \psi+a^{2} \frac{\partial^{2} V}{\partial \phi^{* 2}} \delta \varphi^{*}+a^{2} \frac{\partial^{2} V}{\partial \phi^{*} \partial \phi} \delta \varphi=0 .
$$

Similarly by varying $S_{m}$ with respect to $\Phi$ we obtain the complex conjugate of eqs.(27)-(28). It should be noticed that the first order terms are entirely determined by eqs.(24)-(25), eq.(28) and its complex conjugate. These equations hold for all values of $k$ and, therefore, the range of validity is not limited by the horizon scale. The solutions of these equations will be valid as long as $|\delta \varphi / \phi|$ and $\psi$ are sufficiently small, irrespective of $k$.

The background solution is also established by eq.(17), eq.(27) and its complex conjugate. The fact that eq.(18) and eq.(26) are automatically satisfied is due to the Bianchi identities.

The conservation of the current $J$ leads to a set of two additional equations. For the background part of the solution, we get the conservation with respect to the conformal time of the bosonic charge

$$
\Xi=\frac{i a^{2}}{2}\left(\dot{\phi}^{*} \phi-\dot{\phi} \phi^{*}\right)
$$

and for the first order term the expression

$$
\partial_{\eta} \xi=a^{2} k^{2}\left(\phi^{*} \delta \varphi-\phi \delta \varphi^{*}\right),
$$


where

$$
\xi=4 a^{2} \psi\left(\dot{\phi}^{*} \phi-\dot{\phi} \phi^{*}\right)+a^{2}\left(\dot{\phi}^{*} \delta \varphi-\dot{\phi} \delta \varphi^{*}+\phi \dot{\delta} \varphi^{*}-\phi^{*} \dot{\delta} \varphi\right) .
$$

For very large wavelengths $\xi$ is also a conserved quantity, since then the right hand side of eq.(30) vanishes up to first order.

\section{The Newtonian regime}

In this section, we derive the Jeans wavenumber starting from the general relativistic equations. This way, we avoid the so-called "Jeans swindle" [17]. The gauge invariant formalism is very well adapted to this problem, since in the longitudinal mode $(B=E=0) \psi$ is just the Newtonian potential.

To obtain the Jeans wavenumber we have to derive the dispersion relation. We consider a potential with a mass and a quartic self-interaction term. The Newtonian regime is realized when the expansion of the universe can be neglected and the horizon size is much larger than the wavelength of the perturbation (i.e. $k \gg h$ and $h \sim \epsilon$, where $\epsilon \ll 1$ ). With these conditions it follows from eq.(17) that

$$
k^{2} \gg 8 \pi G \dot{\phi} \dot{\phi}^{*}
$$

and

$$
k^{2} \gg 8 \pi G a^{2} V \equiv 8 \pi G a^{2}\left[m^{2} \phi \phi^{*}+\lambda\left(\phi \phi^{*}\right)^{2}\right] .
$$

We obtain two conditions from eq.(17), because the right hand side of this equation is a sum of two positive terms. ¿From eq.(18) we have $\dot{h} \ll k^{2}$ and thus $|\ddot{a} / a| \ll k^{2}$. We have now to find the solution for the background in the Newtonian regime. Inserting

$$
\phi=f / a
$$

into eq.(27) we get

$$
\ddot{f}+f\left[a^{2} m^{2}+2 \lambda f f^{*}-\dot{h}-h^{2}\right]=0 .
$$

Imposing

$$
a^{2} m^{2}+2 \lambda f f^{*} \gg\left|\dot{h}+h^{2}\right|=|\ddot{a} / a|
$$

- which means, that for an oscillation period of $\phi, a$ can be considered as a constant - eq.(35) can be solved and we get

$$
f=f_{0} \exp (i \omega \eta)
$$


where

$$
\omega=\sqrt{a^{2} m^{2}+2 \lambda f_{0}^{2}}
$$

and $f_{0}$ is an integration constant. Using eq.(34), we have

$$
\phi=b_{0} \exp (i \omega \eta)
$$

with $b_{0}=f_{0} / a$, which within our approximations is constant. Hence, eqs.(32)-(33) and eq.(36) can be rewritten as

$$
\begin{aligned}
& k^{2} \gg 8 \pi G b_{0}^{2} a^{2}\left(m^{2}+2 \lambda b_{0}^{2}\right), \\
& k^{2} \gg 8 \pi G b_{0}^{2} a^{2}\left(m^{2}+\lambda b_{0}^{2}\right), \\
& \left|\frac{\ddot{a}}{a}\right| \ll a^{2} m^{2}+2 \lambda a^{2} b_{0}^{2} .
\end{aligned}
$$

To obtain the dispersion relation we perform the following expansion

$$
\begin{gathered}
\Phi \equiv\left(b_{0}+\frac{b_{1} Y}{a}\right) \exp \left(i \omega \eta+i \frac{\varphi Y}{a}\right)=\phi+\frac{Y}{a}\left(b_{1}+i b_{0} \varphi\right) e^{i \omega \eta}+\mathcal{O}(2), \\
\psi=\frac{\Psi}{a} .
\end{gathered}
$$

As next, we rewrite eq.(24) and eq.(28) in terms of $\Psi, b_{1}$ and $\varphi$. Eq.(28) will then split into a real and an imaginary part. With the following expansion

$$
b_{1}=b_{10} \exp (\Omega \eta), \quad \varphi=\varphi_{10} \exp (\Omega \eta), \quad \Psi=\Psi_{10} \exp (\Omega \eta)
$$

and using the above mentioned Newtonian approximations, we get a set of 3 equations

$$
\begin{gathered}
16 \pi G a^{2} b_{0} b_{10}\left(m^{2}+2 \lambda b_{0}^{2}\right)+8 \pi G b_{0}^{2} \Omega \omega \varphi_{10}-k^{2} \Psi_{10}=0, \\
b_{10}\left(\Omega^{2}+k^{2}+4 \lambda a^{2} b_{0}^{2}\right)-2 \Omega \omega b_{0} \varphi_{10}-2 \Psi_{10} a^{2} b_{0}\left(m^{2}+2 \lambda b_{0}^{2}\right)=0, \\
2 \omega \Omega b_{10}+\varphi_{10} b_{0}\left(\Omega^{2}+k^{2}\right)+4 \Psi_{10} b_{0} \omega \Omega=0 .
\end{gathered}
$$

After some algebraic manipulations, we find

$$
\left[\begin{array}{ccc}
16 \pi G b_{0} \omega^{2} & 8 \pi G b_{0}^{2} \omega \Omega & -k^{2} \\
0 & -\frac{\Omega b_{0}}{2 \omega}\left(4 \omega^{2}+\Omega^{2}+k^{2}+4 \lambda a^{2} b_{0}^{2}\right) & a_{22} \\
0 & 0 & a_{33}
\end{array}\right]\left[\begin{array}{c}
b_{10} \\
\varphi_{10} \\
\Psi_{10}
\end{array}\right]=0
$$


with

$$
a_{22}=\frac{1}{16 \pi G b_{0} \omega^{2}}\left(-32 \pi G b_{0} \omega^{4}+k^{2} \Omega^{2}+k^{4}+4 \lambda a^{2} b_{0}^{2} k^{2}\right)
$$

and

$$
\begin{aligned}
a_{33}= & \frac{1}{32 \pi G b_{0} a^{2} \omega^{2} k^{2}}\left[-64 \pi G b_{0}^{2} \omega^{4} k^{2}+2 k^{6}+8 \lambda a^{2} b_{0}^{2} k^{4}\right. \\
& \left.+\Omega^{2}\left(2 k^{4}+64 \pi G b_{0}^{2} \omega^{2}+2 k^{2}\right)\left(4 \omega^{2}+\Omega^{2}+k^{2}+4 \lambda a^{2} b_{0}^{2}\right)\right] .
\end{aligned}
$$

$a_{33}=0$ is the dispersion relation, which is the required condition for having a non-trivial solution of eq.(49). The Jeans wavenumber, corresponding to the solution of the dispersion relation with $\Omega=0$, is given by

$$
k_{j}^{2}=2 a^{2} b_{0}\left[\sqrt{\lambda^{2} b_{0}^{2}+8 \pi G\left(m^{2}+2 \lambda b_{0}^{2}\right)^{2}}-\lambda b_{0}\right] .
$$

For $\lambda=0$ we obtain the same result as in Ref. 12. Notice that, since we have taken the conformal time, we have an over-all $a^{2}$ factor. For $\lambda \neq 0$, we disagree with the expression for the Jeans wavenumber given by eq.(31) in Ref.[12]. This is due to the fact that eq.(1) in Ref. [12] is not compatible with a quartic self-interaction term. Indeed, for the mass-less complex scalar field with $\lambda \neq 0$, we expect to have a non-vanishing Jeans wavenumber as it is the case for our result, given by eq.(52), but not for the corresponding eq.(31) in Ref.[12]. Only disturbances whose wavenumber is smaller than the Jeans wavenumber $k_{j}$ can grow. Hence, the knowledge of the Jeans wavelength $\lambda_{j}=2 \pi / k_{j}$ gives a rough idea of the size of the objects which can be formed by gravitational collapse. What we do not know, however, is the rate at which the perturbations will grow.

\section{Classical perturbations of a complex scalar field}

In this section we analyze the time evolution of the scalar mode fluctuations in a fully general relativistic way. We will consider the long wavelength and the short wavelength limits separately. To solve the Einstein eqs.(24)-(25), we first define the complex valued function $U(\eta)$ as the solution of the following differential equation

$$
\dot{U}+h U=-4 \pi G \dot{\phi}^{*} \delta \varphi .
$$

$1 k_{j}^{2} \simeq \frac{8 \pi G m^{2}\left(m^{2}+|\lambda|^{2} a^{2}\right)}{|\lambda|^{2}}$ 
For $|\delta \varphi| \lesssim\left|\dot{\phi}^{*}\right| / 4 \pi G$ the complex scalar field perturbation is solution of the constraint eqs. (24)-(25) if and only if the Bardeen potential $\psi$ and $U$ satisfy the following equations

$$
\begin{gathered}
\psi=U+U^{*}+\frac{k_{\psi}}{a}, \\
\ddot{U}+2\left(h-\frac{\ddot{\phi}^{*}}{\dot{\phi}^{*}}\right) \dot{U}+\left(k^{2}+2 \dot{h}-2 h \frac{\ddot{\phi}^{*}}{\dot{\phi}^{*}}\right) U=-\frac{k_{\psi}}{2 a}\left(k^{2}+\dot{h}-h^{2}\right),
\end{gathered}
$$

the r.h.s of the last equation represents a source term and $k_{\psi}$ is a real integration constant. In fact the term $k_{\psi} / a$ in eq.(54) is a solution of the corresponding homogeneous eq.(25), namely $\dot{\psi}+h \psi=0$. In the real scalar field case there is no source term [10]. To simplify the notation we omit

to write the explicit $k$ dependence of $U$. Setting $U=\frac{\dot{\phi}^{*} u}{a}$, eq.(55) can be rewritten as

$$
\ddot{u}+k^{2} u+\left(\dot{h}-h^{2}+\frac{\dddot{\phi}^{*}}{\dot{\phi}^{*}}-2 \frac{\ddot{\phi}^{* 2}}{\dot{\phi}^{* 2}}\right) u=-\frac{k_{\psi}}{2 \dot{\phi}^{*}}\left(k^{2}+\dot{h}-h^{2}\right) .
$$

With $g=\frac{h}{a \dot{\phi}^{*}}$ and using the background equation we find

$$
\ddot{u}+k^{2} u+\left(\frac{32 \pi G i \Xi}{a^{2}}-\frac{\ddot{g}}{g}\right) u=-\frac{k_{\psi}}{2 \dot{\phi}^{*}}\left(k^{2}+\dot{h}-h^{2}\right) .
$$

By solving this last equation we get immediately $U$. Using eq.(54) and eq.(53) we obtain $\psi$ and $\delta \varphi$, respectively.

In the analysis of eq.(57) we will restrict ourselves to long and short wavelength perturbations in the inflationary and the oscillatory phase of the background solution.

\subsection{Perturbations during inflation}

We consider inflation generated by the potential

$$
V=m^{2} \phi \phi^{*}+\lambda\left(\phi \phi^{*}\right)^{2},
$$

for which during inflation $|\phi|^{2} \dot{\vartheta}$ is asymptotically zero [8], where we have set $\phi=|\phi| e^{i \vartheta}$. ¿From eq.(29) we get

$$
a^{2}|\phi|^{2} \dot{\vartheta}=\Xi
$$

where $\Xi$ is the constant bosonic charge. Hence, inflation must start with a small value of $\Xi / a^{2}$. On the separatrix, where inflation occurs, $\Xi / a^{2}$ decays 
exponentially, whatever the values of $m$ and $\lambda$ are. As a consequence eq.(57) reduces to

$$
\ddot{u}+k^{2} u-\frac{\ddot{g}}{g} u=-\frac{k_{\psi}}{2 \dot{\phi}^{*}}\left(k^{2}+\dot{h}-h^{2}\right) .
$$

Moreover, we see from eq.(59) that for small values of $\Xi$ the inflationary phase lasts longer. Eq. (60) is actually valid not only for the inflationary stage but also when $\Xi / a^{2}$ is negligible and $\left|\dot{\phi}^{*}\right| \gtrsim 4 \pi G|\delta \varphi|$.

During inflation we have for $\lambda \neq 0$ and $\lambda=0$, respectively

$$
\ddot{g} / g \simeq \frac{a^{2} V_{\phi \phi^{*}}}{2} \simeq-\frac{3}{2} a^{2} H_{t}=-\frac{3}{2}\left(\dot{h}-h^{2}\right),
$$

and

$$
\ddot{g} / g \simeq \frac{2}{3} a^{2} V_{\phi \phi^{*}} \simeq-2 a^{2} H_{t}=-2\left(\dot{h}-h^{2}\right),
$$

where we have denoted $\frac{\partial V}{\partial \phi \partial \phi^{*}}$ by $V_{\phi \phi^{*}}$ and introduced $H=\frac{1}{a} \frac{d a}{d t} \equiv \frac{a_{t}}{a}$ with $t$ being the real time defined as $d t=a d \eta$ ( $H_{t}$ means derivative of $H$ with respect to real time). This result can be easily obtained using the inflationary background solutions (see [8]). Since inflation occurs only when $V \gg \phi_{t} \phi_{t}^{*}$, we have $\left|H_{t}\right| \ll H^{2}$. This means that $\ddot{g} / g \ll a^{2} H^{2}$ and $\ddot{g} / g$ increases exponentially. Hence the time interval, where $k \simeq \ddot{g} / g$, is very short.

For $k \ll \ddot{g} / g$ - the long wavelength perturbations - the integration of eq. (60) gives

$$
u=k_{1} g+k_{2} g \int \frac{d \eta}{g^{2}}-g \int \frac{1}{g^{2}}\left[\int \frac{\eta}{k_{\psi}\left(\dot{h}-h^{2}\right)} \dot{\phi}^{*} d \tilde{\eta}\right] d \eta
$$

with $k_{1}$ and $k_{2}$ being complex integration constants. Using the background equations and the fact that the phase of the complex scalar field is constant, we can rewrite eq. 63) as

$$
u=\frac{k_{2} e^{-2 i \vartheta}}{8 \pi G \dot{\phi}^{*}} \frac{d}{d \eta}\left[\frac{1}{a} \int^{\eta} a^{2} d \tilde{\eta}\right]-\frac{k_{\psi}}{2 \dot{\phi}^{*}} .
$$

In this last expression the second integration constant has been absorbed in the integral. It follows that for this case the second term in eq.(64), which is due to the source term (see eq.(55)), gives no contribution as we will see below. With eq. (64) we obtain for the gauge invariant metric perturbation

$$
\begin{aligned}
\psi & =\frac{2}{a} \operatorname{Re}\left(\tilde{k_{2}}\right)\left[\frac{1}{a} \int^{\eta} a^{2} d \tilde{\eta}\right]^{\cdot}=2 \operatorname{Re}\left(\tilde{k_{2}}\right) \frac{d}{d t}\left[\frac{1}{a} \int^{t} a d \tilde{t}\right] \\
& =2 \operatorname{Re}\left(\tilde{k_{2}}\right)\left[1-\frac{H}{a} \int^{t} a d \tilde{t}\right] \\
& =2 \operatorname{Re}\left(\tilde{k_{2}}\right)\left(\left[H^{-1}\right]^{\cdot}-\left[H^{-1}\left[H^{-1}\right]^{\cdot}\right]^{\cdot}+\left[H^{-1}\left[H^{-1}\left[H^{-1}\right]^{\cdot}\right]^{\cdot}\right]-\cdots\right)
\end{aligned}
$$


and during the inflationary stage

$$
\psi \simeq-2 R e(\tilde{k}) \frac{H_{t}}{H^{2}}
$$

where $\tilde{k_{2}}=k_{2} e^{-2 i \vartheta} / 8 \pi G$ and $R e$ means the real part. For the gauge invariant field perturbation we get

$$
\delta \varphi=-\frac{k_{2} \dot{\phi}^{*}}{4 \pi G a^{2}} \int^{\eta} a^{2} d \tilde{\eta}=-\frac{k_{2} \phi_{t}^{*}}{4 \pi G a} \int^{t} a d \tilde{t}
$$

and during inflation

$$
\delta \varphi \simeq-\frac{k_{2} \phi_{t}^{*}}{4 \pi G H}
$$

For $k \gg \ddot{g} / g$ - the short wavelength perturbations - eq.66) can be integrated and we obtain

$$
\begin{gathered}
u=\gamma_{1} \cos k \eta+\gamma_{2} \sin k \eta+\frac{\cos k \eta}{k} \int^{\eta} \frac{k_{\psi} k^{2}}{2 \dot{\phi}^{*}} \sin k \tilde{\eta} d \tilde{\eta} \\
-\frac{\sin k \eta}{k} \int^{\eta} \frac{k_{\psi} k^{2}}{2 \dot{\phi}^{*}} \cos k \tilde{\eta} d \tilde{\eta} .
\end{gathered}
$$

As a consequence,

$$
\begin{gathered}
\psi=2 \operatorname{Re}\left[\frac{\dot{\phi}}{a}\left(\gamma_{1} \cos (k \eta)+\gamma_{2} \sin (k \eta)\right)\right]+\frac{k_{\psi}}{a}\left(1-\frac{3 k^{2}}{4 M_{p}^{2} \lambda+3 k^{2}}\right) \\
\simeq 2 \operatorname{Re}\left[\phi_{t}\left(\gamma_{1} \cos \left(k \int^{t} \frac{d \tilde{t}}{a}\right)+\gamma_{2} \sin \left(k \int^{t} \frac{d \tilde{t}}{a}\right)\right)\right] \\
\delta \varphi=-\frac{1}{4 \pi G}\left\{\frac{k}{a}\left[\gamma_{2}^{*} \cos (k \eta)-\gamma_{1}^{*} \sin (k \eta)\right]\right. \\
\left.+\frac{\ddot{\phi}^{*}}{a \dot{\phi}^{*}}\left[\gamma_{1}^{*} \cos (k \eta)+\gamma_{2}^{*} \sin (k \eta)\right]\right\}
\end{gathered}
$$

where $\gamma_{1}, \gamma_{2}$ are complex integration constants and $M_{p}^{2}=1 / 8 \pi G$. Notice, that also for this case the term proportional to $k_{\psi}$ does practically not affect $\psi$. During inflation for a perturbation wavelength smaller than the Hubble radius $-k^{2} \gg a^{2} H^{2} \simeq \ddot{\phi}^{*} / 2 \dot{\phi}^{*}$ — we find

$$
\delta \varphi \simeq-\frac{k}{4 \pi G a}\left[\gamma_{2}^{*} \cos (k \eta)-\gamma_{1}^{*} \sin (k \eta)\right] .
$$


The slow-rolling approximation, required for having a sufficiently long inflationary stage, leads to a slowly variation of the mean value of $\psi$. The behavior of $\delta \varphi$ is governed by the $1 / a$ factor, which decreases rapidly. It follows, as expected, that the short wavelength fluctuations of the scalar field are smeared out.

We consider an initial perturbation with wavelength smaller than the Hubble radius $\left(k^{2} \gg a^{2} H^{2} \gg \ddot{g} / g\right)$, which will be outside it at the end of inflation $\left(k^{2} \ll a^{2} H^{2}\right)$. Indeed there are wavelengths that fulfill these conditions, since $a^{2} H^{2}$ increases exponentially (see Fig. 1). At the end of inflation, the evolution of the gauge invariant metric potential is given by eq.(65). Later on, when the universe is dominated by relativistic particles, the scale factor is a power-law function $a \propto t^{\mu}$. Hence, the Hubble radius increases more rapidly than the fixed comoving wavelength. The metric perturbation can thus re-enter inside the Hubble radius and induce fluctuations on the ordinary matter. These fluctuations would be the source for the anisotropies in the CMB, which have been measured by COBE [1].

At the Hubble radius crossing, with eq.(65), the metric perturbation is given by

$$
\psi=2 \operatorname{Re}\left(\tilde{k_{2}}\right) \frac{1}{\mu+1} .
$$

We can now use the relation between $\tilde{k}$ and $\delta \varphi$ at the time $t_{c}$, defined such that $k=\ddot{g} / g$, and substitute it into the last equation. We get

$$
\psi=-R e\left(\frac{\delta \varphi}{\phi_{t}^{*}} H e^{-2 i \vartheta}\right)_{t=t_{c}} \frac{1}{\mu+1} .
$$

There is an amplification of the metric perturbation between the time it leaves the Hubble radius and the time it re-enters. Using the asymptotic solutions for the inflationary stage this amplification can be derived analytically. As an example for $\lambda=0$ we obtain

$$
\frac{\psi_{\text {in }}}{\psi_{\text {out }}}=\frac{m^{2} t_{c}^{2}}{3(\mu+1)}
$$

This generalizes the result of previous investigations for the real scalar field case [11]. In eq.(74), the gravitational potential still depends on the value of the complex scalar field perturbation at the time $t=t_{c}$. In order to be able to put constraints on the parameters of the model, by comparing with the observed CMB anisotropies, one should be able to express $\psi$ as a function of the background quantities only. To achieve this one has, as for the real 
scalar case [18], to quantize the complex scalar field. However, this task is not as simple as for the real scalar field, since in the lagrangian there is an additional interaction term between the real and the complex part of the scalar field. We plan to come back on this issue in a future publication.

\subsection{Perturbations during the oscillatory phase}

After the inflationary stage the background solution goes through an oscillatory phase. This corresponds in the phase portrait to winding around a focus point $A$ for which $\phi=\dot{\phi}=0$. For a massive complex scalar field the asymptotic behavior of the background solutions around the point $A$ is discussed in Ref. [8]. Expressing the asymptotic behavior as function of the conformal time, we have

$$
\begin{aligned}
& a=3 a_{0} m^{2} \eta^{2}, \\
& \phi=\frac{2 M_{p}}{\sqrt{3} a_{0} m^{3} \eta^{3}}\left[\cos \vartheta_{30} \cos \left(a_{0} m^{3} \eta^{3}-\eta_{1}\right)\right. \\
& \left.+i \sin \vartheta_{30} \cos \left(a_{0} m^{3} \eta^{3}-\eta_{2}\right)\right], \\
& \dot{\phi}=-\frac{2 \sqrt{3} M_{p}}{\eta}\left[\cos \vartheta_{30} \sin \left(a_{0} m^{3} \eta^{3}-\eta_{1}\right)\right. \\
& \left.+i \sin \vartheta_{30} \sin \left(a_{0} m^{3} \eta^{3}-\eta_{2}\right)\right],
\end{aligned}
$$

for $\eta \rightarrow \infty$. $a_{0}, \eta_{1}, \eta_{2}, \vartheta_{30}$ are dimensionless integration constants. Due to the following scaling behavior

$$
\begin{aligned}
& a\left(a_{0}^{-1 / 3} \eta\right)=a_{0}^{1 / 3} a(\eta) \\
& \phi\left(a_{0}^{-1 / 3} \eta\right)=\phi(\eta)
\end{aligned}
$$

and

$$
\dot{\phi}\left(a_{0}^{-1 / 3} \eta\right)=a_{0}^{1 / 3} \dot{\phi}(\eta)
$$

of the background equations, we can restrict the analysis to $a_{0}=1$.

For the study of the first order fluctuations eq.(56) can apriori only be used as long as $\left|\dot{\phi}^{*}\right| \gtrsim 4 \pi G|\delta \varphi|$. $\left|\dot{\phi}^{*}\right|$ is a damped oscillating function which has its minimum on an oscillation period when

$$
b \equiv \cos ^{2} \vartheta_{30} \sin ^{2}\left(m^{3} \eta^{3}-\eta_{1}\right)+\sin ^{2} \vartheta_{30} \sin ^{2}\left(m^{3} \eta^{3}-\eta_{2}\right)
$$


is minimum. If $b$ is large enough (such as $b / \eta>|\delta \varphi|$ ) then eq.(56) will be valid for a large number of oscillations. On the contrary, if the minimum of $b$ is very small, then there will be for each oscillation period two time intervals where eq.(56) is no longer expected to be valid. One can easily see that the minimum of $b$ vanishes for $\vartheta_{30}=n \pi / 2$ or $\left(\eta_{2}-\eta_{1}\right)=n \pi$, where $n$ is an integer. These conditions correspond to a vanishing bosonic charge $\Xi$. Indeed, inserting eqs.(76)-(78) in eq.(29), we find

$$
\Xi=2 \Xi_{\max } \cos \vartheta_{30} \sin \vartheta_{30} \sin \left(\eta_{2}-\eta_{1}\right),
$$

where $\Xi_{\max }=18 M_{p}^{2} m$ is the maximum bosonic charge. The maximum value of all possible minima of $b$ is reached for $\vartheta_{30}=\pi / 4+n \pi / 2$ and $\eta_{2}-\eta_{1}=$ $\pi / 2+l \pi$ ( $l$ being an integer). For these and only these values of $\vartheta_{30}$ and $\left(\eta_{2}-\eta_{1}\right), b=1 / 2$ and $|\Xi|$ is maximum. One can show that by increasing the minimum of $b$ also the absolute value of the bosonic charge increases. Following these lines, we see that the time interval, where eq.(56) is valid, increases with $|\Xi|$. Hence, we will restrict our analysis to $\Xi \sim \Xi_{\max }$.

With eqs.(76)-(78) we can easily show that

$$
\left|\frac{\dddot{\phi}^{*}}{\dot{\phi}^{*}}-2 \frac{\ddot{\phi}^{* 2}}{\dot{\phi}^{* 2}}\right| \simeq \frac{9 m^{6} \eta^{4}}{b}\left((b-2)^{2}-2 \frac{\Xi^{2}}{\Xi_{\max }^{2}}\right) .
$$

The right hand side can never be zero for any value of $b$. It follows that

$$
\left|\dot{h}-h^{2}\right| \ll\left|\frac{\dddot{\phi}^{*}}{\dot{\phi}^{*}}-2 \frac{\ddot{\phi}^{* 2}}{\dot{\phi}^{* 2}}\right| .
$$

Thus, eq.(56) reduces to

$$
\ddot{u}+k^{2} u-\frac{\ddot{p}}{p} u=-\frac{k_{\psi}}{2 \dot{\phi}^{*}}\left(k^{2}+\dot{h}-h^{2}\right),
$$

where $p=1 / \dot{\phi}^{*}$. There are three regimes to study. The first is given by the condition $k^{2} \gg|\ddot{p} / p| \gg\left|\dot{h}-h^{2}\right|$, the second by $|\ddot{p} / p| \gg k^{2} \gg\left|\dot{h}-h^{2}\right|$ and the third by $|\ddot{p} / p| \gg\left|\dot{h}-h^{2}\right| \gg k^{2}$.

For the short wavelength perturbations $-k^{2} \gg|\ddot{p} / p|-$ eq.(69) can again be used. Using that for $\Xi \sim \Xi_{\max }$

$$
\phi \simeq \mp i \frac{\sqrt{2} M_{p}}{\sqrt{3} m^{3} \eta^{3}} e^{ \pm i\left(m^{3} \eta^{3}-\eta_{0}\right)}
$$

and

$$
\dot{\phi} \simeq \frac{\sqrt{6} M_{p}}{\eta} e^{ \pm i\left(m^{3} \eta^{3}-\eta_{0}\right)}
$$


we get that

$$
\begin{aligned}
\psi & \simeq 2 \operatorname{Re}\left[\frac{\dot{\phi}}{a}\left(\gamma_{1} \cos (k \eta)+\gamma_{2} \sin (k \eta)\right)\right]+\frac{k_{\psi}}{a}+\mathcal{O}\left(1 / \eta^{4}\right) \\
& \simeq 2 \operatorname{Re}\left[\phi_{t}\left(\gamma_{1} \cos \left(k \int^{t} \frac{d \tilde{t}}{a}\right)+\gamma_{2} \sin \left(k \int^{t} \frac{d \tilde{t}}{a}\right)\right)\right]+\frac{k_{\psi}}{a}
\end{aligned}
$$

and $\delta \varphi$ is given by eq.(71), since it can be shown that the inhomogeneous solution is of order $1 / \eta^{6}$. The different signs in eqs.(84)-(85) depend on the integers $n$ and $l$. We see that the dominant term of $\psi$ decreases like $1 / a$. With eq.(71) we have

$$
\delta \varphi \simeq-\frac{1}{4 \pi G} \frac{\ddot{\phi}^{*}}{a \dot{\phi}^{*}}\left[\gamma_{1}^{*} \cos (k \eta)+\gamma_{2}^{*} \sin (k \eta)\right],
$$

since in general

$$
\begin{aligned}
\frac{\ddot{\phi}^{*}}{a \dot{\phi}^{*}}=\frac{m}{2 b}[ & \cos ^{2} \vartheta_{30} \sin \left(2 m^{3} \eta^{3}-2 \eta_{1}\right) \\
& \left.+\sin ^{2} \vartheta_{30} \sin \left(2 m^{3} \eta^{3}-2 \eta_{2}\right)-i \frac{\Xi}{\Xi_{\max }}\right]
\end{aligned}
$$

and $k / a$ is a decreasing function. It follows that the real and the imaginary part of $\delta \varphi$ oscillate around a constant value.

For the long wavelength case $-k^{2} \ll\left|\dot{h}-h^{2}\right| \ll|\ddot{p} / p|-u$ is now given by eq.(63), where we have to substitute $g$ by $p$. It is easy to see that for large $t$

$$
\psi=\frac{\operatorname{Re}\left(k_{1}\right)}{a}+\frac{1}{t}\left(k_{3}+k_{3}^{*}\right)+\mathcal{O}\left(\frac{1}{t^{2}}\right)
$$

where

$$
k_{3}=2 k_{2} M_{p}^{2}\left(\sin ^{2} \vartheta_{30}-\cos ^{2} \vartheta_{30}+2 i \sin \vartheta_{30} \cos \vartheta_{30}\right) .
$$

It should be noticed that for $\Xi \sim \Xi_{\max }, k_{3}+k_{3}^{*}$ is a very small real number. In the long wavelength regime the metric perturbation is essentially driven by the expansion. For the computation of $\delta \varphi$ we use also eq. (53) and obtain

$$
\delta \varphi=-\frac{k_{2}}{4 \pi G a} \dot{\phi}^{*}=-\frac{k_{2}}{4 \pi G} \phi_{t}^{*}
$$

For this case the source term gives only a negligible contribution to $\delta \varphi$, and $\psi$ gets another term proportional to $1 / a$, which is absorbed in its homogeneous solution. 
In the intermediate case, where $\left|\dot{h}-h^{2}\right| \ll k^{2} \ll|\ddot{p} / p|$, $u$ is given by

$$
u=k_{1} p+k_{2} p \int \frac{d \eta}{p^{2}}-p \int \frac{1}{p^{2}}\left[\int^{\eta} \frac{k_{\psi} k^{2}}{2 \dot{\phi}^{*}} d \tilde{\eta}\right] d \eta
$$

After some computations we see that the solution of $\psi$ is also given by eq.(89). For the complex scalar field perturbation instead we have

$$
\delta \varphi=-\frac{k_{2}}{4 \pi G a} \dot{\phi}^{*} \mp \frac{i k_{\psi} k^{2}}{36 m^{3} a} \dot{\phi} .
$$

Hence, the first order perturbation of the scalar field decays in the long wavelength and in the intermediate regime, whereas it oscillates around a constant value in the short wavelength. Therefore, during the oscillatory phase there can be no formation of gravitational seeds, like boson stars, by a massive scalar field with sufficiently large bosonic charge.

Using the background asymptotic solution [8], we obtain the analytic behavior of the metric and the complex perturbations. They are reported in Table 1. For all the other cases the solutions have to be found numerically.

\section{$5 \quad$ Numerical results}

To complete our analysis, we study the evolution of the perturbations after inflation, which implies that the bosonic charge $\Xi$ is close to zero.

As a first step we integrate the background equations from an initial singularity point $S$, where the equation of state is given by $\varepsilon^{(0)}=\mathbf{T}^{(\mathbf{0})}$ (stiff matter) and the asymptotic behaviors of $\phi, \dot{\phi}$ and $H$ are as follows 8

$$
\begin{gathered}
\phi=M_{p} \sqrt{3}\left[C+i \frac{1}{3} \ln \left(t / t_{0}\right)\right] \\
\dot{\phi}=M_{p} \sqrt{3}\left[-\frac{C m^{2} t}{2}+\frac{i}{3 t}\right], \\
H=\frac{1}{3 t}
\end{gathered}
$$

for $t \rightarrow 0^{+}, C$ and $t_{0}$ being integration constants. It should be noticed that the bosonic charge $\Xi$ depends directly on the choice of the value of $C$ and $t_{0}$. Hence, starting from $S$ we can freely fix the value of $\Xi$. In order to have a long inflationary phase we must choose a small value of $\Xi$, which is 
achieved for instance by setting $C=1$ and $t_{0}=100$. With these values the background starts with a stiff matter regime and, after an inflationary phase ( $h$ exponentially increases, see Fig. 1), ends in the oscillatory phase ( $h$ oscillates around a slowly decreasing curve, see Fig. 1). For a massive complex scalar field inflation is also less effective when $\Lambda$ increases (indeed, the maximum of $h$ decreases with $\Lambda$, see Fig. 1). This, because the potential becomes less flat and as a consequence the slow-roll approximation, needed for inflation to occur, is no longer well satisfied.

As a next step we integrate numerically eq.(26), eq.(28) and its complex conjugate using the background solution. We control our numerical computation by looking if eqs.(24)-(25) propagate. Moreover, we check if we recover the analytic solutions described in section $₫$.

The Bardeen potential $\psi$ behaves as predicted during inflation and remains, up to a smooth oscillation, constant after inflation. This behavior is valid for any value of the parameters $k, m$ and $\lambda$.

For a long period during inflation $|\delta \varphi|$ decreases and thus, at first glance, does not behave as expected from our analytic analysis (see Fig. 3). For the cases $\Lambda=0.1$ and $m=0$ with $\lambda=1$ we even get that during inflation $|\delta \varphi|$ is only decreasing. This may seem to be in contradiction with the analytic results obtained in section 4.1. However, this is not true since we do not start the integration of the background right away from the singularity point, where inflation occurs. If we did so, we would have obtained the expected analytic behavior for $|\delta \varphi|$. The $|\delta \varphi|$ trajectory which we get by the analytic treatment, as given in Table 1, should be interpreted as an "attractor" for solutions. Indeed, if inflation is powerful (large e-fold number), the time at which the perturbation is generated becomes unimportant and $|\delta \varphi|$ will rapidly approach the behavior found analytically. For the cases where inflation is not so powerful (see Fig. 1), the shape of the fluctuations of $|\delta \varphi|$ will depend more on the details of the background solution from which they were produced. That is exactly what we find in Fig. 3. After inflation the long wavelength perturbations of $|\delta \varphi|$ oscillate.

For an initial fluctuation $\delta \varphi$, which lies inside the Hubble radius, the exponential damping is so effective, that at the end of inflation $\delta \varphi$ practically vanishes, as can be seen in Fig. 5. This is valid whatever the values of $\lambda$ and $m$ are.

We notice that, to check numerically the analytic behavior for the situation where $\Xi \sim \Xi_{\max }$, is more difficult, since one has to deal with numerical instabilities. 


\section{Concluding Remarks}

In this paper we studied the perturbation of the coupled Einstein-KleinGordon equations for a complex scalar field in different regimes. We derived analytically the time evolution of long and short wavelength perturbations during inflation. Moreover, we established that in order to have a long period of inflation the bosonic charge must be close to zero. After inflation the perturbation of the metric remains constant during the oscillatory phase.

If the complex scalar field has a large bosonic charge or the values of the parameters entering in the potential are such that the slow-roll approximation does not apply, then it turns out that inflation can not have taken place or lasted only for a short period. Hence, the background passes directly in the oscillatory phase. We showed that in the massive case, for $\Xi \sim 0$ or $\Xi \sim \Xi_{\text {max }}$, the perturbations of the scalar field decay or, at best, stay constant and thus no gravitational seeds, like boson stars, can form during this epoch. Constraints on the parameters of the scalar field potential coming from the measured CMB anisotropies can only be put once a thorough study of the quantized perturbations will be done. However, since inflation is essentially driven by one component of the complex field, we do not expect significant differences to occur on the constraints and thus on the physical implications as compared to the real scalar field case 10 .

We also derived the Jeans wavenumber for the Newtonian regime starting from the general relativistic equations.

\section{Acknowledgments}

We thank N. Deruelle and N. Straumann for very useful discussions.

\section{References}

[1] C.L. Bennett et al. , Astrophys. J. 464, L1 (1996)

[2] P. Jetzer, Phys. Rep. 220, 163 (1992)

[3] A. Liddle and M. Madsen, Int. J. of Mod. Phys. D1, 101 (1992)

[4] V. A. Belinsky, L. P. Grishchuk, I. M. Khalatnikov and Ya. B. Zeldovich, Phys. Lett. B155, 232 (1985)

[5] V. A. Belinsky, L. P. Grishchuk, Ya. B. Zeldovich and I. M. Khalatnikov, Sov. Phys. JETP 62195 (1985) 
[6] V. A. Belinsky and I. M. Khalatnikov, Sov. Phys. JETP 66441 (1987)

[7] T. Piran and R.M. Williams, Phys. Lett. B163, 331 (1985)

[8] D. Scialom and P. Jetzer, Phys. Rev. D51, 5698 (1995)

[9] N. Deruelle, C. Gundlach and D. Langlois, Phys. Rev. D45, 3301 (1992) N. Deruelle, C. Gundlach and D. Polarski, Class. Quantum Grav. 9, $1511(1992)$

[10] V.F. Mukhanov, H.A. Feldman and R.H. Brandenberger, Phys. Rep. 215203 (1992)

[11] A. Starobinsky, Phys. Lett. 117B, 175 (1982)

S. Hawking, Phys. Lett. 115B,295 (1982)

A. Guth and S. Y. Pi, Phys. Rev. Lett. 49, 1110 (1982)

J. Bardeen, P. Steinhardt and M. Turner, Phys. Rev. D28, 679 (1983)

R. Brandenberger, Rev. Mod. Phys. 57, 1 (1985)

R. Brandenberger, Int. J. Mod. Phys. A2, 77 (1987)

[12] M. Yu. Khlopov, B. A. Malomed and Ya. B . Zeldovich, Mon. Not. R. astr. Soc. 215, 575-589 (1985)

[13] H. Kodama and M. Sasaki, Prog. Th. Phys. Supp. 78, 1 (1984)

[14] C. W. Misner, K. S. Thorne and J. A. Wheeler, Gravitation (Freemann 1973)

[15] R. Durrer and N. Straumann, Helv. Phys. Act. 61, 1027 (1988)

[16] J. Bardeen, Phys. Rev. D 22, 1882 (1980)

[17] S. Weinberg, Gravitation and Cosmology, John Wiley and Sons (1972)

[18] V.F. Mukhanov, Sov. Phys. JETP 68, 1297 (1988) 


\section{Caption}

Table 1:

Summary of the analytic solutions during the inflationary and the oscillatory phases for the metric and the complex perturbations.

Figure 1:

$h=a H$ as function of the cosmological time $t$ for three different values of $\Lambda=\frac{\lambda M_{p}^{2}}{m^{2}}$ and for the case $m=0, \lambda=1 . \lambda$ is the coupling constant of the quartic self-interaction term, $m$ is the mass of the scalar field and $M_{p}$ is the Planck mass. For the massive cases $h(t)$ is in units of $m\left(m^{-1}\right)$ and for the massless case $h(t)$ is in units of $M_{p}\left(M_{p}^{-1}\right)$.

Figure 2:

Bardeen potential $\psi$ for the zero mode $(k=0)$ as a function of the cosmological time $t$ for different values of $m$ and $\lambda$. The units of $t$ are the same as in Fig. 1.

Figure 3:

Norm of $|\delta \varphi|$ for the zero mode as a function of the cosmological time $t$ for different values of $m$ and $\lambda$. For the massive cases (massless case) $|\delta \varphi|$ is in units of $m\left(M_{p}\right)$. For the units of $t$ see Fig. 1.

Figure 4:

Same as for Fig. 2 but with $k=60000$

Figure 5:

Norm of $|\delta \varphi|$ for $k=60000$ and $\Lambda=0$ as a function of the cosmological time $t$. For the units of $|\delta \varphi|$ and $t$ see Fig. 3 and Fig. 1. 


\begin{tabular}{|l|l|l|}
\hline \multicolumn{3}{|c|}{ During Inflation } \\
\hline & $\lambda=0$ & $\lambda \neq 0$ \\
\hline $\begin{array}{l}k^{2} \gg \ddot{g} / g \\
\text { (short wavelength) }\end{array}$ & $\begin{array}{l}\psi: \text { oscillates } \\
\delta \varphi: \text { exponentially } \\
\text { damped }\end{array}$ & $\begin{array}{c}\psi: \text { oscillates in an expo- } \\
\text { nentially decaying } \\
\text { envelope } \\
\delta \varphi: \text { exponentially } \\
\text { damped }\end{array}$ \\
\hline $\begin{array}{l}k^{2} \ll \ddot{g} / g \\
\text { (long wavelength) }\end{array}$ & $\begin{array}{l}\psi \propto \frac{1}{t^{2}} \\
|\delta \varphi| \propto-\frac{1}{t} \\
(t \rightarrow-\infty)\end{array}$ & $\begin{array}{l}\psi \propto \exp \left(4 M_{p} \sqrt{\lambda / 3} t\right) \\
|\delta \varphi| \propto \exp \left(2 M_{p} \sqrt{\lambda / 3} t\right) \\
(t \rightarrow-\infty)\end{array}$ \\
\hline
\end{tabular}

\begin{tabular}{|l|l|}
\hline \multicolumn{2}{|c|}{ During the Oscillatory Phase } \\
\hline & \multicolumn{1}{|c|}{$m \neq 0$ and $\Xi / \Xi_{\text {max }} \approx 1$} \\
\hline $\begin{array}{l}k^{2} \ll\left|\dot{h}-h^{2}\right| \ll \ddot{p} / p \\
\text { and } \\
\left|\dot{h}-h^{2}\right| \ll k^{2} \ll \ddot{p} / p \\
\text { (long wavelength) }\end{array}$ & $\begin{array}{l}\psi \propto 1 / a \propto 1 / t^{2 / 3} \\
|\delta \varphi| \propto \frac{1}{t} \\
(t \rightarrow+\infty)\end{array}$ \\
\hline $\begin{array}{l}k^{2} \gg \ddot{p} / p \\
\text { (short wavelength) }\end{array}$ & $\begin{array}{l}\psi: 2 \text { terms, the first oscillates in a } \frac{1}{t} \text { en- } \\
\text { velope, whereas the second is propor- } \\
\text { tional to } 1 / t^{2 / 3} \text { for } t \rightarrow+\infty\end{array}$ \\
& $\begin{array}{l}\delta \varphi: 2 \text { terms, the first oscillates and the sec- } \\
\text { ond oscillates in a }\left(\frac{1}{t}\right)^{2 / 3} \text { envelope, for } \\
\end{array}$ \\
& $t \rightarrow+\infty$. \\
\hline
\end{tabular}

Table 1 


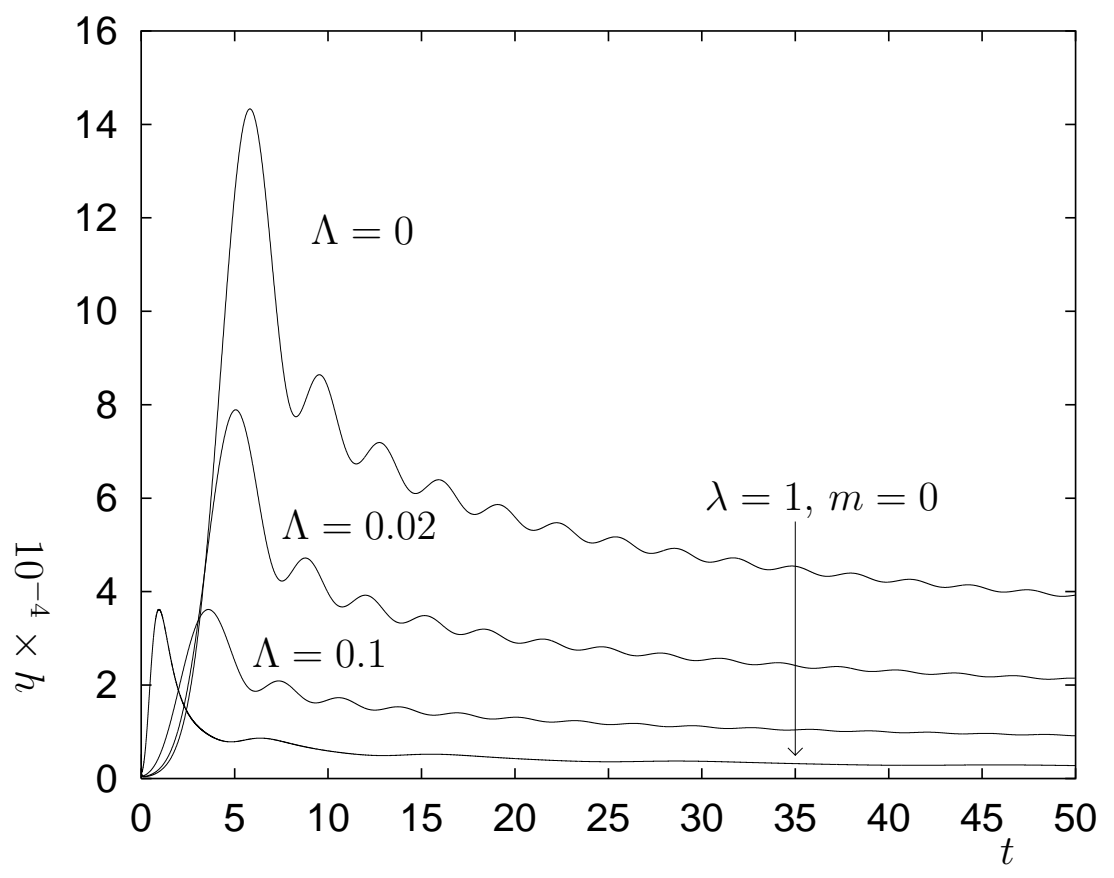

Fig.1 


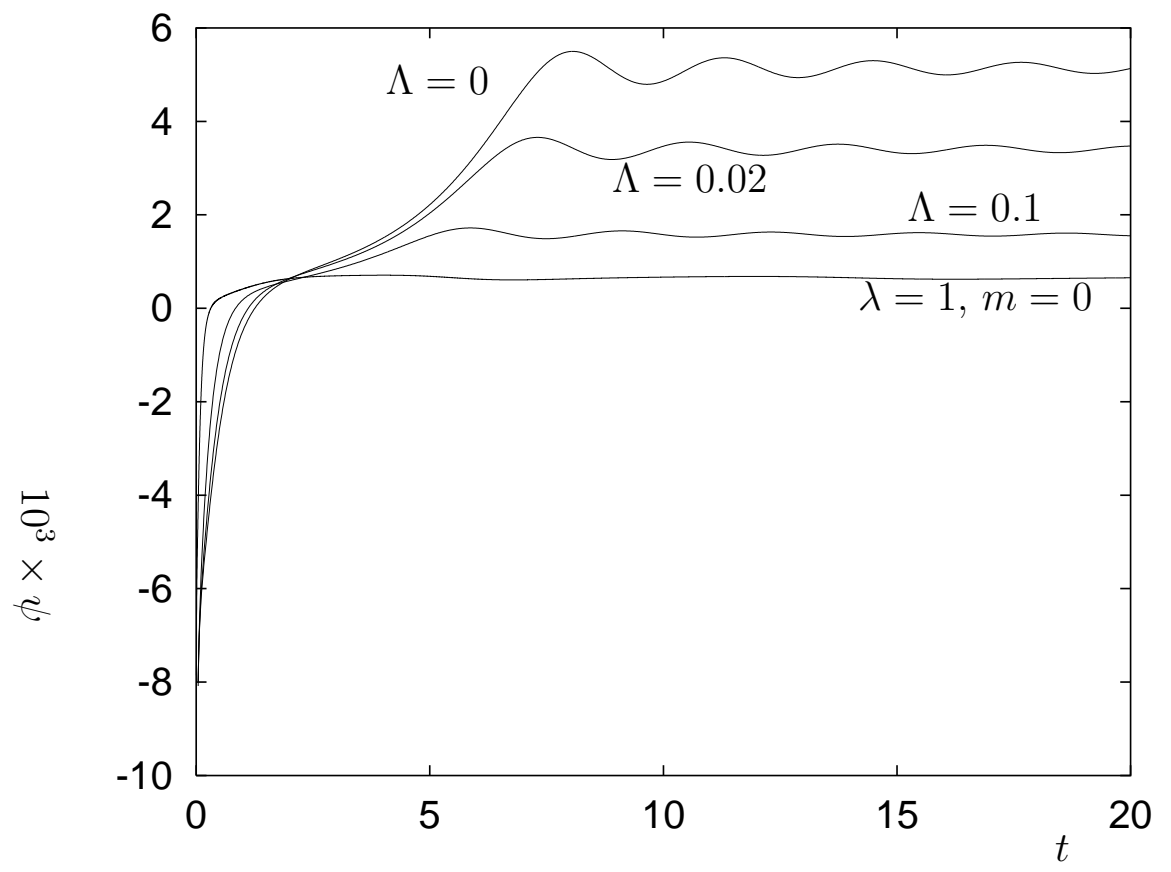

Fig.2 


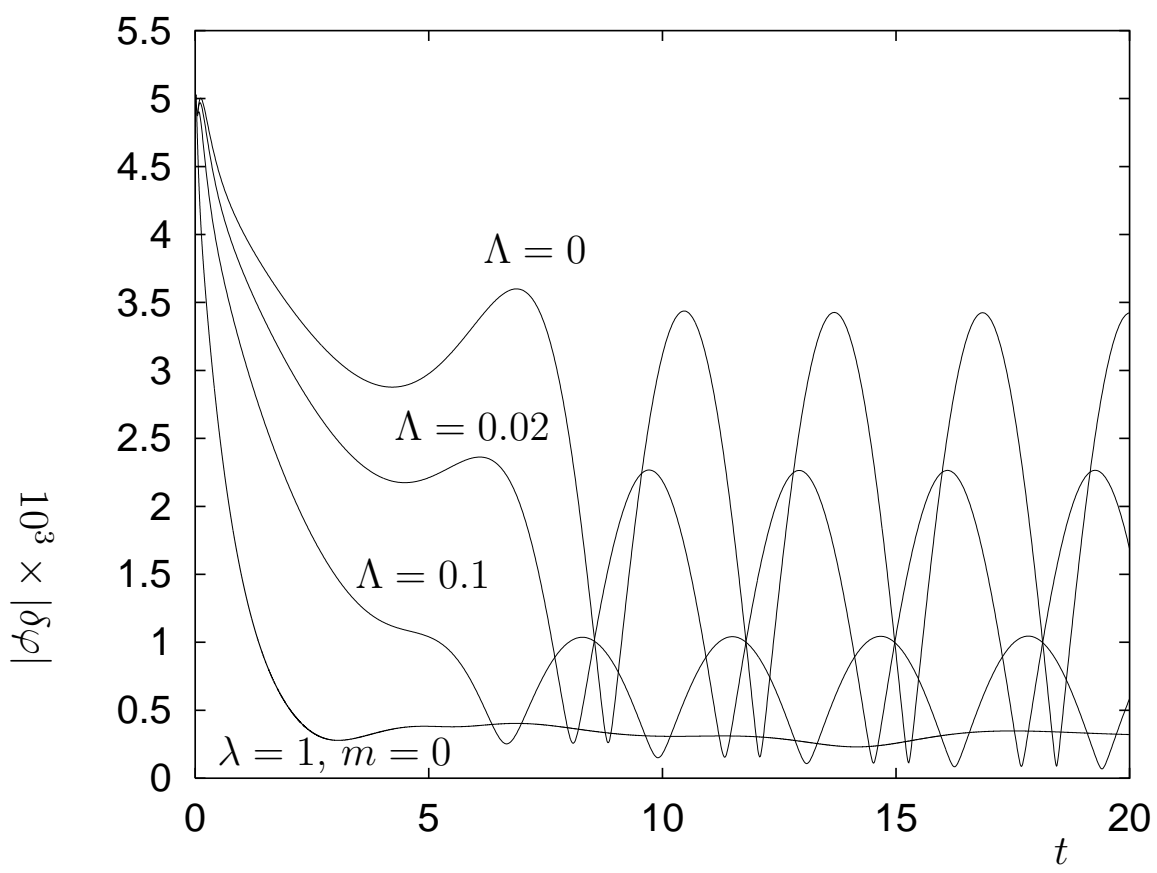

Fig.3 


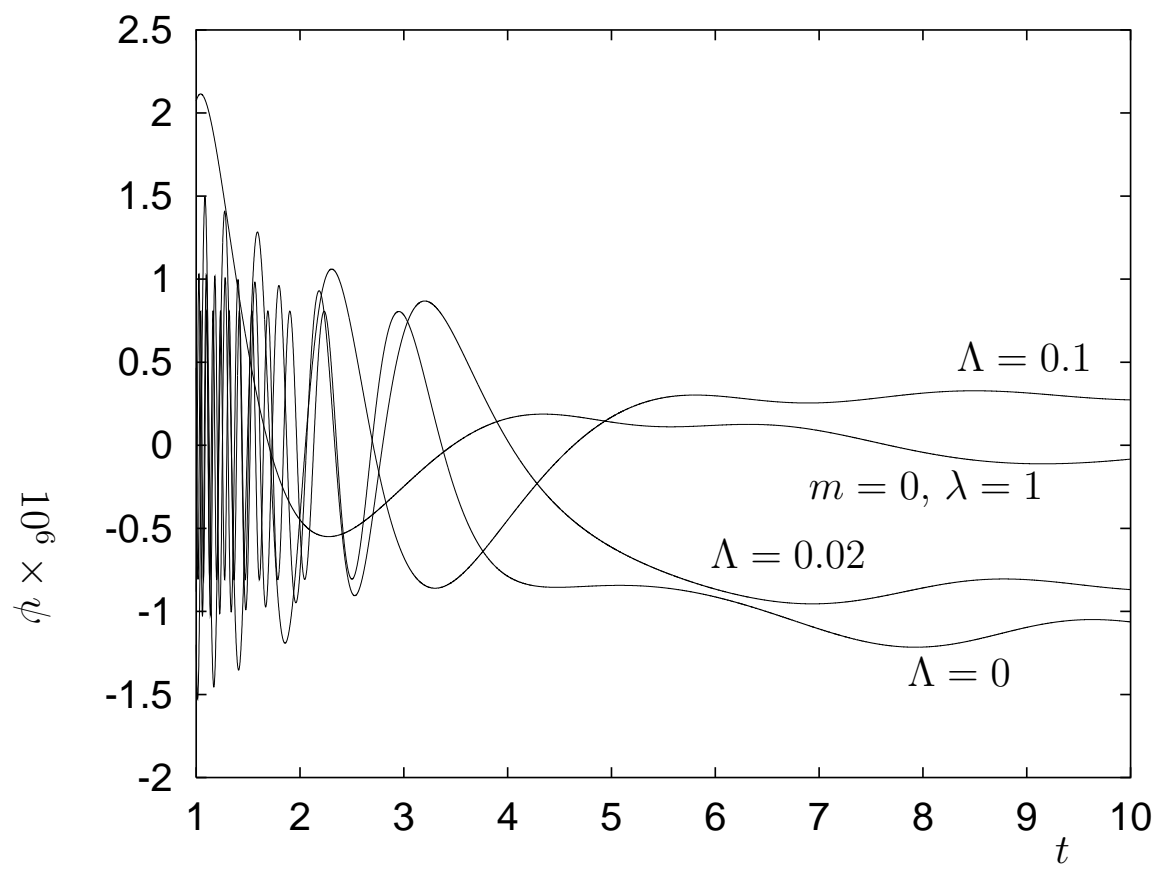

Fig.4 


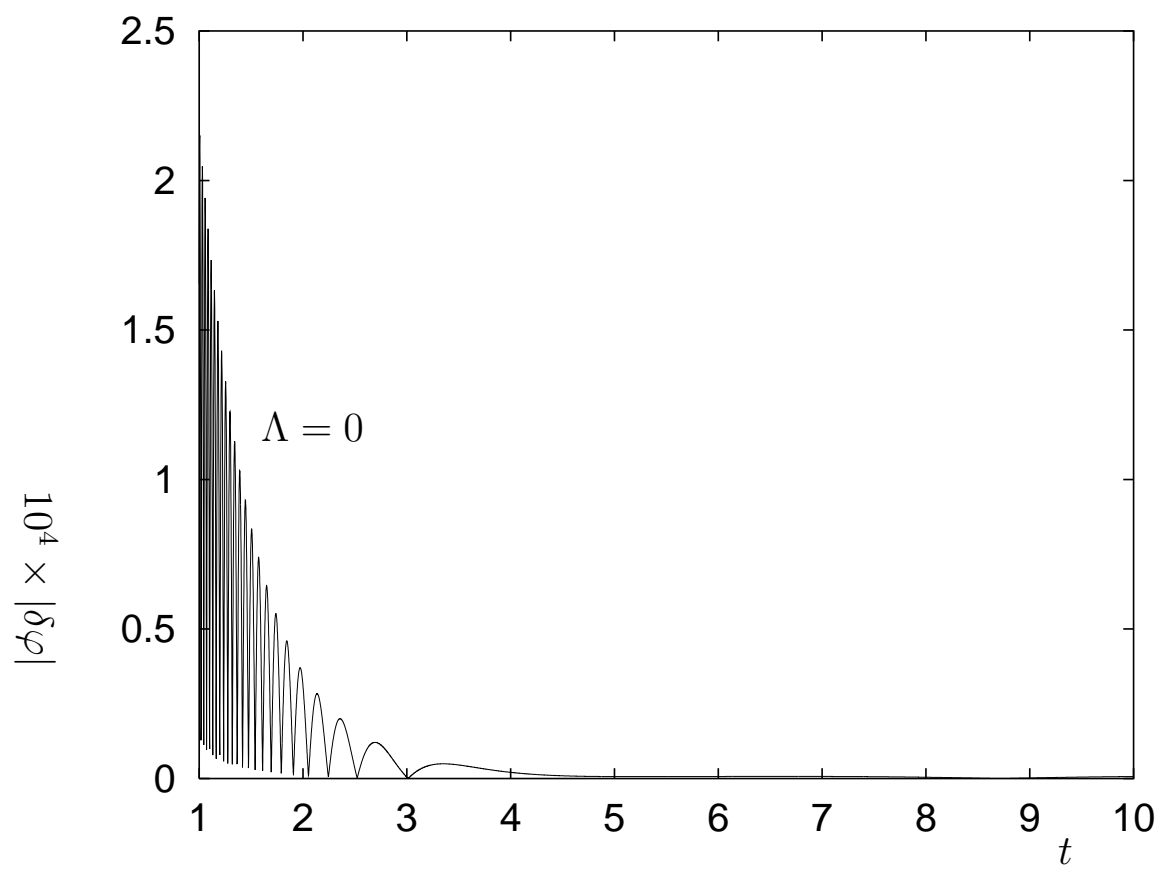

Fig.5 